\title{
Convex Restriction Sets for CBERS-2 Satellite Image Restoration
}

João P. Papa†, Nelson D. A. Mascarenhasł, Leila M. G. Fonsecał, Kamel Bensebaał †UFSCAR- Federal University of São Carlos, São Carlos, Brazil.

\{jpaulo,nelson\}@dc.ufscar.br

¥INPE-National Institute for Space Research, São José dos Campos, Brazil.

\{leila,camel\}@dpi.inpe.br

(v3.0 released September 2004)

The main goal of this work is to develop a new efficient image restoration algorithm based on projections onto convex sets technique (POCS), which uses some a priori information about the images in the form of restriction sets. The proposed convex formulation used in this work is the prototype image contraints, which was obtained by the Modified Inverse Filter, limited amplitude and by the Modified Row-Action Projection (MRAP) algorithm. A simulation experiment was performed using a high resolution IKONOS image, which was blurred and decimated according to CBERS-2 CCD camera specifications. In order to allow quantitative analysis, the ISNR and the Universal Image Quality Index methodologies were applied. An original CBERS-2 CCD image was also used to evaluate the proposed restoration method. The restored images show a good visual performance, which can also be observed by the autocorrelation coefficients, which indicate high frequency enhancement.

Keywords: Image Restoration, POCS, CBERS-2, IKONOS

\section{Introduction}

The amount of satellite imagery has widely increased with the new sophisticated onboard imaging systems. These sensors provide high quality data and allow a more accurate understanding of on ground phenomena.

Among this new generation of satellites, the CBERS project (China-Brazil Earth Resources Satellite) was jointly developed by Brazil and China and its mission was designed to create four satellites to capture high-resolution images of the Earth by using panchromatic and multispectral detectors. The CBERS-2 
satellite is the second Earth observation satellite developed with this cooperation, and it carries three sensors: Wide Field Imager (WFI), High Resolution CCD Camera (CCD) and Infrared Multispectral Scanner (IR-MSS). The CCD Camera has 4 spectral bands and provides images of $113 \mathrm{~km}$ wide strips with sampling rate of 20 meters at nadir Bensebaa et al. (2003).

However, remote sensing images still have limitations. Therefore, the images need to be processed to better reflect its radiometric and geometric quality. One of the radiometric correction techniques is image restoration. Its goal is the reconstruction or recovery of the degraded image using a priori knowledge of the degradation phenomenon. The optical defocussing, combined with the analog to digital converter and any corrections necessary to compensate for internal detector problems results in an image where the effective instantaneous field of view (EIFOV) is different from the nominal resolution value, resulting in a blurring effect.

These distortions can be approximated by a gaussian blur model, which characterizes the effect of the imperfect optical focus of the lens, eletronic filter and detector size. This model is similar to a low-pass filter, which produces an image with blurred appearance. The burring effect, however, can be compensated by a restoration filter that is applied in the image to reduce its blurred appearance.

The problem of satellite image restoration arises from the characteristics of the modulation transfer function (MTF) of optical instruments, either multispectral scanners or imaging spectrometers. In fact, the radiance signal cannot be sampled at the Nyquist frequency (frequency at which the MTF holds the value of 0.5 ), because the heavy tailed MTF would introduce an unacceptable amount of aliasing. Therefore the image is slightly oversampled (typical MTF value at cutoff frequency is 0.2 for multispectral sensors; even lower, e.g., 0.1, for very high resolution panchromatic images). Hence, the images may have a blurred appearance, that is likely to compromise both visual and automated classification tasks. Restoration of multispectral images is crucial because the majority of methods tend to increase the noisiness of the data, thus lowering its radiometric quality, i.e., the SNR.

A large number of image restoration methods have been developed for several applications, like Inverse and Wiener Filter, regularization techniques and MAP techniques. Some works oriented towards remote sensing can be cited: Fonseca et al. (1993) developed the Modified Inverse Filter, which is a regularized version of the Inverse Filter to restore and interpolate Landsat images; $\mathrm{Wu}$ and Schowengerdt (1993) dealt with the restoration of images containing mixed pixels; Bhaskar et al. (1994) tackled the problem of lens defocus and linear motion blur in Space Shuttle images; Reichenbach et al. (1995) described the design of small convolution kernels for the restoration and reconstruction of Advanced Very High Resolution Radiometer (AVHRR) images; 
Boo and Bose (1997) applied image restoration techniques to multispectral images; Jalobeanu et al. (1993) used complex wavelet packets to deconvolve degraded images; Likhterov and Kopeika (2002) dealt with the problem of vibration in images with a differential scheme; Chen and Xanju (1994) restored SAR images using the technique of Independent Component Analysis; Papa et al. (2005) compared two POCS techniques: the Row-Action Projetion Method (RAP) and the Simultaneous Iterative Reconstruction Technique (SIRT) to restore CBERS-1 images.

The POCS method uses a priori knowledge about the image or the imaging system. The key to effectively apply this kind of algorithm is to define the appropriate sets, compute the projection onto these sets, and incorporate the projectors into an image processing algorithm designed to meet some criteria implied by the constraints (Stark 1998).

In that way, the main goal of this work is to design a new efficient image restoration algorithm based on POCS methodology. To the best of our knowledge, this paper is the first application of projections onto convex sets technique for the restoration of remote sensing images.

In order to quantitatively evaluate the results, we used the well known improvement signal to noise ratio, ISNR, and the recent approach Universal Image Quality Index Wang and Bovik (2002). Our work is the first using this metric in this area of knowledge. The remainder of the paper is organized as follows. Section 2 presents the image restoration formulation. Section 3 contains the POCS theory. Section 4 discusses the experimental results and Section 5 provides some conclusions.

\section{Image Restoration}

The image restoration problem reported here is to obtain an estimate of an image $\mathbf{f}$ from its degraded and noisy observation $\mathbf{g}$ which is the result of a linear imaging system modeled by

$$
g=H f+n
$$

where $\mathbf{H}$ is the convolutional degradation operator, denoted here as the block circulant matrix, and $\mathbf{n}$ denotes the additive observation noise (Gonzalez and Woods 2001). Vectors $\mathbf{g}, \mathbf{f}$ and $\mathbf{n}$ correspond to lexicographical ordering of the respective two-dimensional fields by rows, with dimension $M$, and columns, with dimension $N$. Consequently, these vectors and matrix $\mathbf{H}$ have, respectively, $M N x 1$ and $M N x M N$ dimensions. 
The image restoration can be understood as a technique used to correct the distortions produced by the imaging systems. The undesirable effect over the image is detail smoothing and the correction of this problem is based on the sensor caracteristics. Therefore, for each satellite, sensor and spectral band an adjusted filter needs to be used.

\subsection{Modified Inverse Filter}

Equation (1) can be written in the Fourier domain as

$$
G=H_{z} F+N
$$

where $G, F$ and $N$ are the Discrete Fourier Transform (DFT) of the degraded image $g$, the original image $f$ and the additive noise $n$, respectively. Matrix $H_{z}$ is the DFT of the zero padded point spread function, which models the limitations of the sensor. In the absence of noise, Equation (2) can be rewritten as

$$
G=H_{z} F
$$

and an obvious choice for the Inverse Filter $\widehat{I}$ is:

$$
\widehat{I}=\frac{1}{H_{z}} .
$$

However, in the presence of noise, Equation (4) is unstable. The instability arises from the fact that restoration is essentially an ill-conditioned problem.

The Modified Inverse Filter, also called Transfer Function Compensation, approximates the Inverse Filter and at the same time attempts to control the problems associated with it. The idea is to choose a desired function $D$ as the response of the system that would alleviate the ill-conditioning effects (Fonseca et al. 1993):

$$
D=H_{z} W
$$

where $W$ is the restoration filter. The function $D$ should have a better behavior than the function W. A constant value for $D$ yields the Inverse Filter. Once $D$ is selected, $W$ can be estimated. Since we have a separable PSF for directions $X$ and $Y$, also called across-track and along-track, respectively, $W, D$ and $H_{z}$ 
INPE ePrint: sid.inpe.br/ePrint@80/2006/10.24.19.25 v2 2007-06-05

will be treated as 1-D functions. In that way, $W$ can be written as

$$
W(u)= \begin{cases}\frac{D(u)}{H_{z}(u)} & |u| \leq u_{c} \\ 0 & \text { otherwise }\end{cases}
$$

where $u$ is the frequency and $u_{c}$ is the system cut-off frequency.

The desired response $D(u)$ used in this work is

$$
D(u)= \begin{cases}1 & 0 \leq u \leq u_{w} \\ 0.5\left(1+\cos \left[\frac{\pi\left(u-u_{w}\right)}{\left(u_{c}-u_{w}\right)}\right]\right) & u_{w}<u \leq u_{c}\end{cases}
$$

where $u_{w}$ is the frequency for which the Modulation Transfer Function is 0.5 (Fonseca et al. 1993).

\section{POCS Technique}

Some problems can be described in terms of convex sets constraints. POCS (Projections onto Convex Sets) methods can be used to find a common vector $f$ which satisfies these constraints, each of which forms a convex set (Stark 1998). If we suppose that we have a priori information constraints about the image, this common vector $f$ lies in the intersection of all the convex sets

$$
f \in C_{0}=\bigcap_{i=1}^{m} C_{i}
$$

where the $i$ th closed convex set $C_{i} \in \Re$ denotes the $i$ th constraint or a priori knowledge on $f$ and $m$ is the number of those sets.

If the sets $C_{i}(i=1, \ldots, m)$ are closed and convex, and their intersection, $C_{0}$ , is non-empty, the sucessive projections on the sets will converge to a vector that belongs to this intersection.

This vector can be found by alternatively projecting it onto the convex sets $C_{i}$ via corresponding projecting operator $P_{C_{i}}$ as

$$
f^{(k+1)}=P_{C_{m}} P_{C_{m-1}} \ldots P_{C_{1}} f(k),
$$

where $P_{C_{i}}$ means the projection onto convex set $C_{i} \in \Re$ in the $k$ th iteration. The initial guess $f^{(0)}$ can be any vector in $\Re$. 


\subsection{Convex Restriction Sets}

3.1.1 Row-Action Projection - RAP. The image restoration linear model can be described by Equation (10)

$$
g=H f
$$

which is similar to Equation (1), but without the noise term, where each line of $g$ denotes an equation that can be represented by a hyperplane, which is a convex set, and its solution can be found by the POCS method.

If the intersection of these convex sets is non-empty, the main goal is to find this intersection set, which will contain the solution of the restoration problem. The POCS algorithm to obtain the solution of the linear system described by Equation (10) is called Row-Action Projection (RAP) or ART (Algebraic Reconstruction Technique) in tomography, which was initially developed by Kaczmarz in 1937 (Mammone 1992). The POCS method is a generalization of RAP, where the hyperplanes may be substituted by other types of convex sets (Kuo and Mammone 1992). The method converges to the hyperplanes intersection, and the RAP equation is given by

$$
f^{(k+1)}=f^{(k)}+\lambda \frac{g_{p}-h_{p}^{t} f^{k}}{\left\|h_{p}\right\|^{2}} h_{p}
$$

where $\lambda$ is the relaxation parameter, $g_{p}$ is the $p$ th element of vector $g, h_{p}^{t}$ is the $p$ th row of matrix $H$ and $f^{(k+1)}$ is the $f^{(k)}$ projection onto the corresponding hyperplane. The iteration index is related to the equation index by $p=k(\bmod M N)$, indicating that each row is used multiple times in the restoration process. Thus, by definition, the $P_{i} x$ projection of a vector $x$ onto $C_{i}$ set is the point in $C_{i}$ closest to $x$. In that way, the $P_{i} x$ projector on a linear system restriction set, based on Equation (11) is

$$
P_{i} x=P_{C_{R}}=f^{(k)}+\lambda \frac{g_{p}-h_{p}^{t} f^{k}}{\left\|h_{p}\right\|^{2}} h_{p}
$$

The success of RAP algorithm implementation depends on the initial condition, iteration number and relaxation parameter $\lambda$.

Modified $\boldsymbol{R A P}$. Imaging systems are generally designed so that the degradation matrix $H$ is sparse. In addition, this degradation operator is a block Toeplitz matrix in the shift-invariant case and will represent a 2-D linear con- 
volution given by

$$
g(i, j)=\sum_{m} \sum_{n} h(i-m, j-n) \widehat{f}(m, n) .
$$

The sparseness of the matrix $\mathbf{H}$ is due to the fact that the size of the PSF is genererally much smaller than the size of the image.

Tha RAP algorithm given by Equation (11) can be implemented by considering only a subregion of the 2-D image $\widehat{f}$ that is determined by the size of the 2-D support of the PSF. In this case, every row of the matrix $\mathbf{H}$ in Equation (10) contains only $N \mathrm{x} N$ entries, where $N$ denotes the PSF size. Each pixel $g(i, j)$ of the blurred image $g$ corresponds to a specific equation of the set given by Equation (11). Hence, the 2-D formulation of the RAP algorithm can be written as

$\widehat{f}^{(k+1)}(m, n)= \begin{cases}\widehat{f}^{(k)}(m, n)+\lambda \frac{\epsilon(i, j)}{\|h(i, j)\|^{2}} h(i-m, j-m ; i, j), & \text { if } \widehat{f}^{(k)}(m, n) \in S_{h(i, j)}, \\ \widehat{f}^{(k)}(m, n) & \text { otherwise, }\end{cases}$

where

$$
\begin{array}{r}
\epsilon(i, j)=g(i, j)-\sum_{m, n \in S_{h(i, j)}} h(i-m, j-n ; i, j) \widehat{f}^{(k)}(m, n), \\
\|h(i, j)\|^{2}=\sum_{m, n \in S_{h(i, j)}} h(m, n ; i, j)^{2},
\end{array}
$$

and $S_{h(i, j)}$ is the support of the PSF centered at pixel $g(i, j)$. In that way, the Modified RAP (MRAP) algorithm can be implemented as a 2-D convolution. That is, each projection operator is local, requiring only the neighborhood $S_{h(i, j)}$ of the image $\widehat{f}$, at each iteration (Kuo and Mammone 1992). The MRAP projection operator is based on Equation (14), just changing $\widehat{f}^{(k+1)}$ of the image by $P_{C_{M R A P}}$.

3.1.2 Limited Amplitude Restriction Set. This set describes the upper and lower bounds for the image pixels values to be restored. Equation (17) describes this convex set, where $\alpha$ and $\beta$ are the lower and upper bounds respectively and $\Omega$ is the support region of the image:

$$
C_{L A}=\{s: s \in S \text { and } \alpha \leq s(k, l) \leq \beta \forall k, l \in \Omega\},
$$


where $S$ is a Hilbert Space. The projection operator onto the set $C_{L A}$ is described as

$$
P_{C_{L A}} x(k, l)= \begin{cases}\alpha, & \text { if } x(k, l)<\alpha \\ x(k, l), & \text { if } \alpha \leq x(k, l) \leq \beta \\ \beta, & \text { if } x(k, l)>\beta\end{cases}
$$

Since it is known that the values of an image need to be in the interval $[0,255]$, in this work we used the following values: $\alpha=0$ and $\beta=255$.

3.1.3 Prototype Image Restriction Set. This restriction set is based on prototype images, which can be obtained as a result of applying a predetermined operator to the observed image. In this case, the operator and the bound, which limits the variation of the restored image from the prototype, are the two defining values of a prototype constraint.

In that way, a prototype image constraint set can be defined by

$$
C_{P I}=\left\{y:\|q-y\|^{2} \leq \delta\right\},
$$

where $q$ and $y$ are the prototype image and an arbitrary member of $C_{P I}$, respectively, and $\delta$ denotes the bound on the variation of $y$ from the prototype (Sezan and Trussel 1991).

Prototype images are usually obtained from the observed image as result of applying a predetermined operator, i.e., $q=O g$. In this case, the general form described by Equation (19) can be rewritten as

$$
C_{P I}=\left\{y:\|O g-y\|^{2} \leq \delta\right\} .
$$

The bound $\delta$ can be written as

$$
\delta=c \psi,
$$

where $c \geq 0$ is determined by the confidence which we would like to have about the ideal solution in the set described by Equation (19), and $\psi$ is the expected variation given by

$$
\psi=E\left\{y:\|W G-F\|^{2} \leq \delta\right\} .
$$

$W$ is the Modified Inverse Filter, $G$ and $F$ are the degraded and estimated images, respectively. Image $F$ was obtained through the convolution between 
G and the PSF, corresponding to an average filter (Sezan and Trussel 1991).

The projection of an arbitrary vector q onto $C_{P I}$ is given by

$$
y^{*}=C_{P I} q \longleftrightarrow Y^{*} \begin{cases}W G-\sqrt{\delta} \frac{\Delta}{|\Delta|} & \text { if }\left|\Delta^{2}\right|>\delta \\ q & \text { otherwise }\end{cases}
$$

where $\Delta=W G-Q$, and $Q$ is the FT of $q$.

\section{Experimental Results}

\subsection{Simulation Tests}

In order to evaluate the results, the following methodology was used. A panchromatic image from IKONOS satellite (1 meter) was processed by a low pass filter similar to the CBERS-2 CCD MTF. Afterwards, the output image was decimated in such a way that the resulting image exhibited approximately the same pixel spacing as CBERS-2 (20 meters). Finally, an amount of noise was added, to complete the degradation process. We intended that this final image, that we call phantom, to be very similar to a true CBERS-2 image. At the same time, we produced a reference image by resampling the panchromatic image to the same CBERS-2 spatial resolution.

In that way, the IKONOS image used in the experiments covers the area of São José dos Campos,Brazil, shown in Figure (1). This image is $6920 \times 3689,8 \mathrm{bits} /$ pixel. The blurring effect was obtained by applying a bidirectional Gaussian PSF corresponding to CBERS-2 band 3, and by adding zero mean Gaussian noise with $\sigma^{2}=10$, where $\sigma^{2}$ is the noise variance. Figures (2) and (3) display, respectively, the reference and phantom images. The standard deviations used to generate the gaussian function are $\sigma_{x}=23.30$ and $\sigma_{y}=17.66$, corresponding to across-track and along-track satellite directions, respectively. More details about the CBERS-2 radiometric characteristcs can be found in Fonseca et al. (2004).

Although the convergence of the POCS is guaranteed, since the intersection of all sets is non-empty, in pratice we chose to use a stop criterion, given by

$$
\frac{\left\|\widehat{f}^{(n)}-\widehat{f}^{(n-1)}\right\|}{\left\|\widehat{f}^{(n-1)}\right\|} \leq \epsilon
$$

where $\widehat{f}^{(n)}$ corresponds to an estimate of the image at $i$ th step (Stark 1998). 


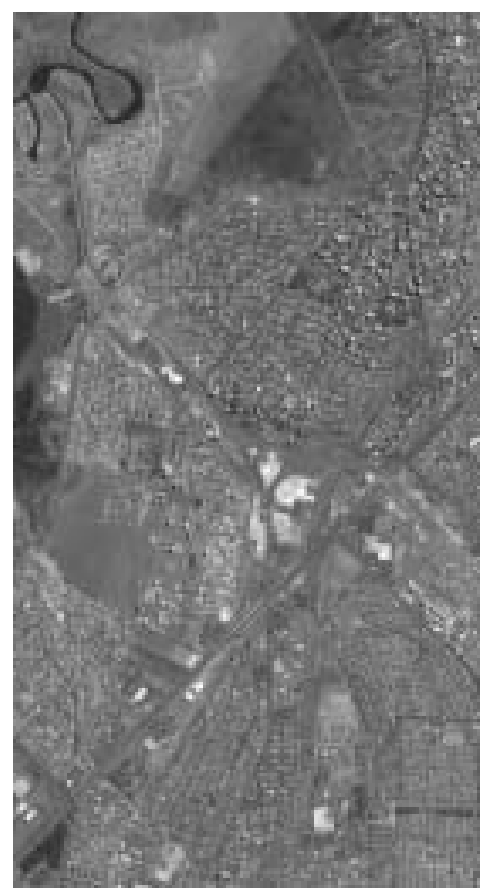

Figure 1. Original IKONOS panchomatic image.

The restored images were evaluated through the ISNR (improvement signal to noise ratio), given by

$$
I S N R=10 \log _{10}\left\{\frac{\sum_{i, j}[g(i, j)-f(i, j)]^{2}}{\sum_{i, j}[\widehat{f}(i, j)-f(i, j)]^{2}}\right\}
$$

where $g(i, j), f(i, j)$ and $\widehat{f}(i, j)$ are, respectively, the degraded, original and restored image. Another approach used to evaluate the restoration algorithm was the Universal Image Quality Index (Wang and Bovik 2002), given by

$$
U=\frac{4 \sigma_{x y} \overline{x y}}{\left(\sigma_{x}^{2}+\sigma_{y}^{2}\right)\left[(\bar{x})^{2}+(\bar{y})^{2}\right]}
$$

where $\bar{x}, \bar{y}, \sigma_{x}^{2}$ and $\sigma_{y}^{2}$ are the average and variance of the original and restored images, respectively, and $\sigma_{x y}$ is the correlation coefficient between $x$ and $y$. The 
INPE ePrint: sid.inpe.br/ePrint@80/2006/10.24.19.25 v2 2007-06-05

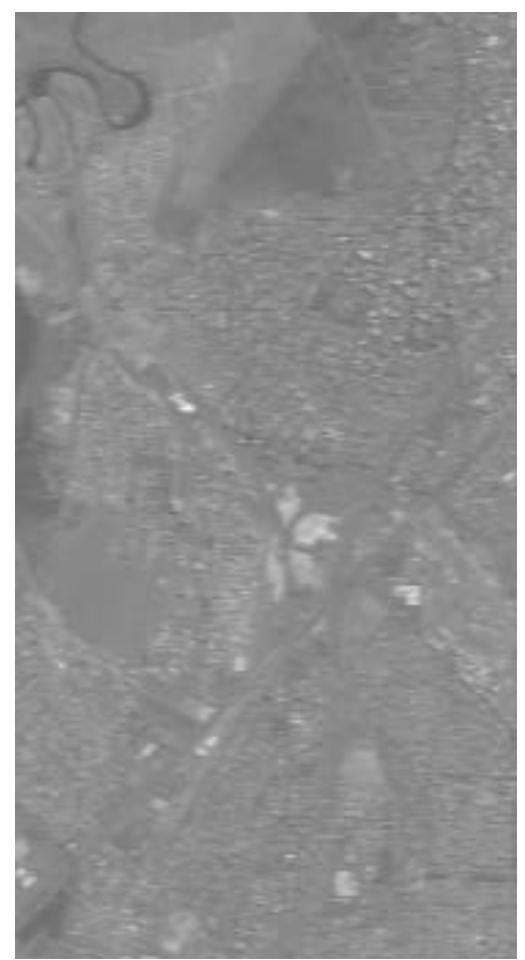

Figure 2. Reference image obtained by decimating the IKONOS panchromatic image to the same CBERS-2 pixel size.

dynamic range of $U$ is $[-1,1]$. The best value (1) is achieved if and only if $y=x$.

The degraded images were restored using the POCS algorithm below:

$$
f^{(k+1)}=P_{C_{L A}} P_{C_{P I}} P_{C_{M R A P}} f(k),
$$

where $P_{C_{L A}}, P_{C_{P I}}$ and $P_{C_{M R A P}}$ are the projections onto the limited amplitude, prototype image and MRAP constraint sets, respectively.

The projection operator $P_{C_{M R A P}}$, described by Equation (14), can be approximated by Inverse Filter or Pseudoinverse solution, when $\mathbf{H}$ is a nonsquare matrix, i.e.,

$$
\lim _{k \rightarrow \infty} f^{(k)}=H^{\dagger} g
$$

where $H^{\dagger}$ denotes the pseudoinverse of $\mathbf{H}$ (Mammone 1992).

We know that the MRAP algorithm is based on Equation (10), which does not consider the noise term, like Equation (1). In that way, there is a need to 


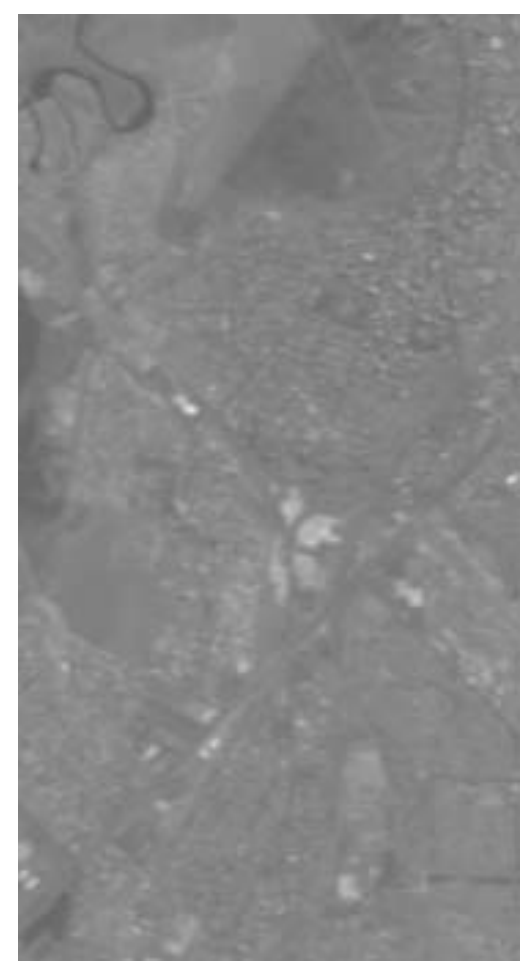

Figure 3. Phantom image obtained by degrading and decimating the IKONOS panchromatic image.

control the effect of ill-conditioning by adding to the algorithm two restriction sets: $P_{C_{P I}}$ and $P_{C_{L A}}$, obtaining Equation (27). Figure (4) shows the result of applying only the MRAP algorithm to the phantom image, which is described by

$$
f^{(k+1)}=P_{C_{M R A P}} f(k) .
$$

Since the linear system given by Equation (1) does not have intersection due to noise term, some artifacts located on the high frequencies regions of the image can be observed. This problem demonstrates the numerical instability that arises from inverse and pseudoinverse filters. Figure (5) displays the restored phantom image using the algorithm given by Equation (27).

The value that maximized ISNR was obtained using the confidence value $c=1$ (Equation 21) and relaxation parameter $\lambda=1.5$ (Equation 11). The obtained value was $I S N R=2.0123$. For the Universal Image Quality Index we obtained $U=0.8808$.

We demonstrate the validity of the proposed algorithm by applying well- 
INPE ePrint: sid.inpe.br/ePrint@80/2006/10.24.19.25 v2 2007-06-05

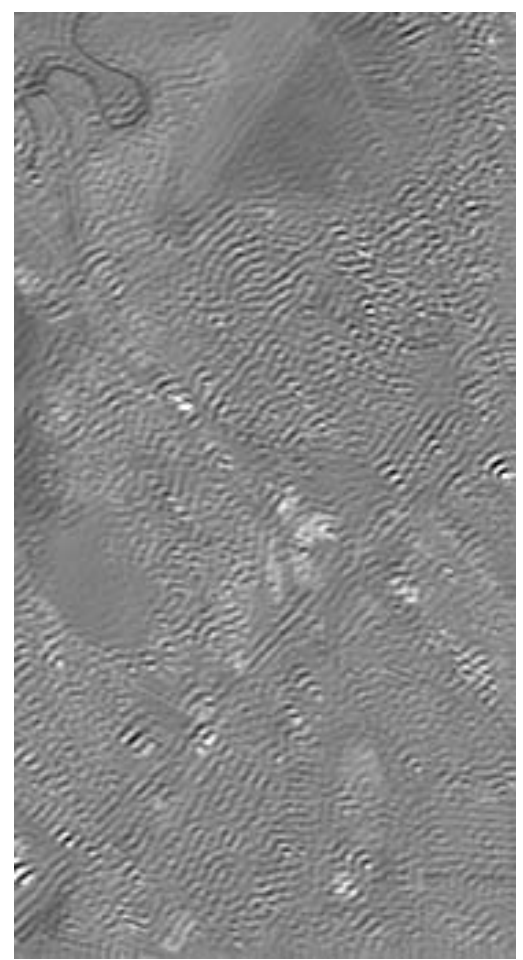

Figure 4. Restored phantom image by applying Equation (29).

known methods to CBERS-2 image: Wiener Filter, Modified Inverse Filter (described in Section 2.1) and POCS algorithm below, named as POCS $S_{I N V}$ :

$$
f^{(k+1)}=P_{C_{P I}} f(k),
$$

where the prototype image used in $P_{C_{P I}}$ was obtained by the Modified Inverse Filter. Table (1) displays the results. It can be observed that the values obtained by $P O C S_{I N V}$ were very close to the Modified Inverse Filter results. The proposed POCS procedure presented better results than those of other tested methods, both in $I S N R$ and $U$.

The experimental results were performed by using a computer with the following characteristics: AMD AThlon XP 2400+ Processor and $512 \mathrm{Mb}$ of RAM DDR memory. The analysis in terms of computational costs (in seconds) is presented in the Table (2).

It was expected that the computational costs of the proposed POCS procedure would be greater than those of the other tested methods, since it is an iterative procedure, compared to one pass procedures of the other methods. 


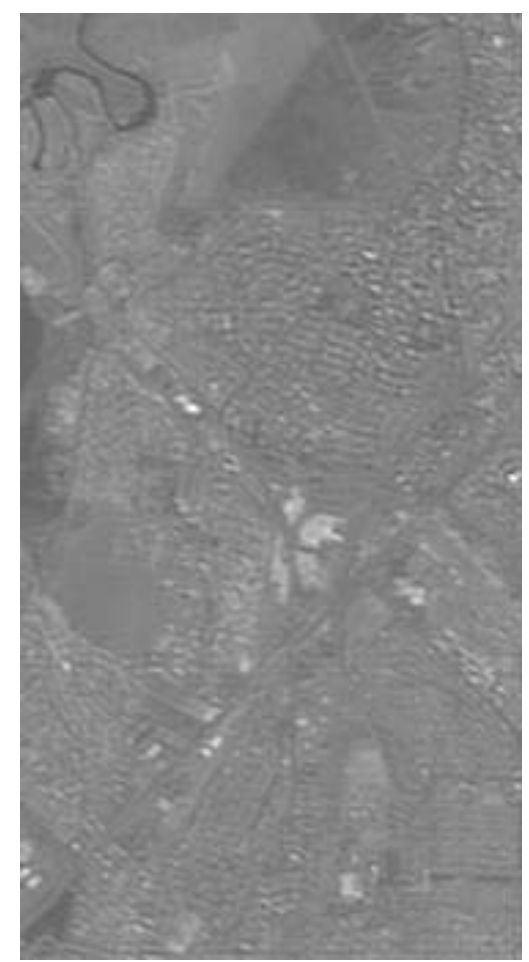

Figure 5. Restored phantom image.

\begin{tabular}{|l|c|c|}
\hline \multicolumn{1}{|c|}{ Algorithm } & ISNR & U \\
\hline Wiener Filter & 1.4456 & 0.7754 \\
\hline Modified Inverse Filter & 1.1334 & 0.7289 \\
\hline POCS $S_{I N V}$ & 1.1298 & 0.7103 \\
\hline Proposed POCS & 2.0123 & 0.8808 \\
\hline
\end{tabular}

Table 1. $I S N R$ and Image Quality Index $(U)$ values for Wiener Filter, Modified Inverse Filter, POCS INV and the proposed POCS procedure applied to phantom image.

\subsection{Real Tests}

Finally, the POCS algorithm given by Equation (27) was applied to an image acquired from CBERS-2 CCD camera. The parameters used in the restoration process to model the bidirecional PSF were $\sigma_{x}=23.30$ and $\sigma_{y}=17.66$, corresponding to across-track and along-track satellite directions, respectively Fonseca et al. (2004). Figures (6) and (7) display, respectively, the original CBERS-2 CCD and restored images. The CBERS-2 image had its contrast enhanced for display purposes only. 
INPE ePrint: sid.inpe.br/ePrint@80/2006/10.24.19.25 v2 2007-06-05

\begin{tabular}{|l|c|}
\hline \multicolumn{1}{|c|}{ Algorithm } & Computational Time \\
\hline Wiener Filter & $1.33 \mathrm{~s}$ \\
\hline Modified Inverse Filter & $1.01 \mathrm{~s}$ \\
\hline$P O C S_{I N V}$ & $5.33 \mathrm{~s}$ \\
\hline Proposed POCS & $84.32 \mathrm{~s}$ \\
\hline
\end{tabular}

Table 2. Displays computational time of Wiener Filter, Modified Inverse Filter, POCS INV and the proposed POCS procedure applied to phantom image.

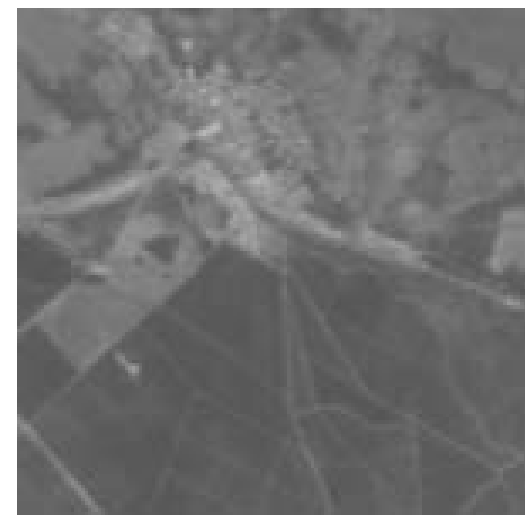

Figure 6. Original CBERS-2 CCD image.

The image quality improvement can be observed visually or through the autocorrelation coefficients, which indicate high frequency enhancement.We noticed that the curve decreases faster for the restored than for the original CBERS-2 image. The normalized autocorrelation coefficients, in directions $x$ and $y$, were computed using the equations below (Gonzalez and Woods 2001):

$$
\gamma_{x}(k)=\frac{A_{x}(k)}{A_{x}(0)},
$$

where

$$
A_{x}(k)=\frac{1}{M(N-k)} \sum_{x=1}^{M} \sum_{y=1}^{N-k} f(x, y) f(x, y+k)
$$

and

$$
\gamma_{y}(k)=\frac{A_{y}(k)}{A_{y}(0)},
$$


where

$$
A_{y}(k)=\frac{1}{(M-k) N} \sum_{x=1}^{M-k} \sum_{y=1}^{N} f(x, y) f(x+k, y)
$$

The variables $M$ and $N$ are the image dimensions.

Figures (8) and (9) display, respectively, the original and restored image normalized autocorrelation coefficients, in both directions, $x$ and $y$.

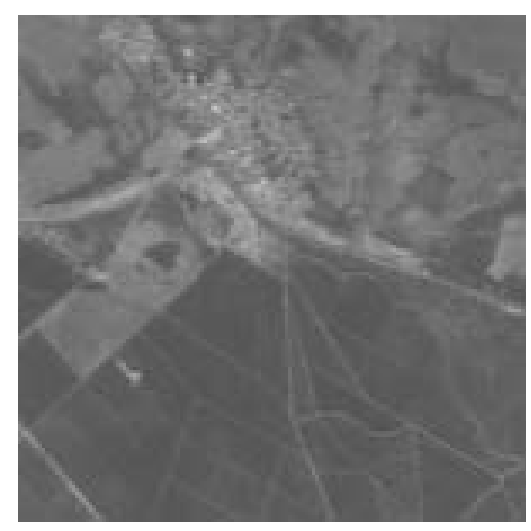

Figure 7. Restored CBERS-2 CCD image.

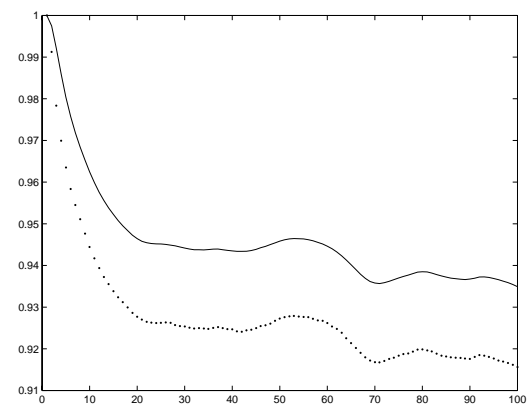

Figure 8. Normalized autocorrelation coefficients for $x$ direction. The continuous and dotted lines are, respectively, the coefficients of original and restored CBERS-2 images. 


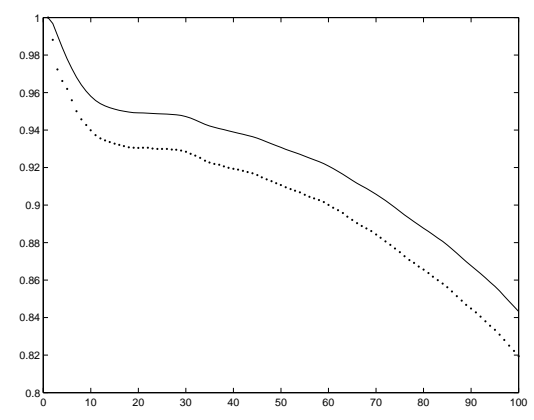

Figure 9. Normalized autocorrelation coefficients for $y$ direction. The continuous and dotted lines are, respectively, the coefficients of original and restored CBERS-2 images.

\section{Conclusions}

We presented a new efficient image restoration method based on POCS technique. We considered the case where the prototype images are obtained from the observed image via a predetermined operator, which is the Modified Inverse Filter. In order to allow quantitative evaluation, a phantom image was obtained by decimating and blurring the high resolution IKONOS image with the CBERS-2 CCD camera specifications. A POCS algorithm using the proposed filter, the limited amplitude and the Modified Row-Action Projection constraints was applied to the phantom image and observed CBERS-2 CCD image (band 3). The results obtained with low levels for $c$ and $\lambda$ were better, with fast convergence (10 iterations). The ISNR and Universal Image Quality Index values obtained by the proposed methodology were better than those of other tested methods. The real experiment showed good visual performance, which can also be observed through their autocorrelation coefficients. Our main contributions are the first application of POCS techniques for remote sensing image restoration and the use of the Universal Quality Index in this area of knowledge.

\section{Acknowlegments}

The first author was supported by a CAPES scholarship. This project was also supported by FAPESP Thematic Project 2002/07153-2. We would like to thank Dr. Hermann Kux from National Institute for Space Research, INPE, for providing the IKONOS images. 


\section{REFERENCES}

Bensebaa K., Banon G.J.F. and Fonseca, L.M.G., Spatial Resolution Estimation of CBERS-1 CCD Imaging System. Conference Proceedings of Applied Computing Workshop at INPE, 205-210, 2003.

Bhaskar R., Hite J., Pitts D.E, An Iterative Frequency-Domain Technique to Reduce Image Degradation Caused by Lens Defocus and Linear Motion Blur. International Geoscience and Remote Sensing Symposium, IGARSS94, vol. 4, 2522-2524, 1994.

Boo, K.J., Bose, N.K., Multispectral image restoration with multisensors. IEEE Transactions on Geoscience and Remote Sensing, vol. 35, no. 05, 1160-1170, 1997.

Chen C.H., Xianju W., A Novel Theory of SAR Image Restoration and Enhancement with ICA. International Geoscience and Remote Sensing Symposium, IGARSS04, vol. 06, 3911-3914, 1995.

Fonseca, L.M.G., Prasad, G.S.S.D, Mascarenhas, N.D.A, Combined Interpolation-Restoration of Landsat Images through FIR Filter Design Tehniques. International Journal of Remote Sensing, vol. 14, no. 13, 25472561, 1993.

Fonseca, L.M.G., Ponzoni, F., Cartaxo,R, Radiometric Quality Assessment of CBERS-2. INPE Technical Report APPL-06-1994, http://www.obt.inpe.br/cbers/documentos/appl_06_2004.pdf, 2004.

Gonzalez R.C., Woods, R. E., Digital Image Processing. Prentice-Hall, 2nd Edition, 2001.

Hunt B.R., The Application of Constrained Least Squares Estimation to Image Restoration by Digital Computer. IEEE Transactions on Computing, vol. C-22, no. 9, 805-812, 1973.

Jalobeanu A., Blanc-Feraud L., Zerubia J., Satellite Image Deconvolution Using Complex Wavelet Packets. International Conference on Image Processing, ICIP 2000, vol. 3, 809-813, 2000.

Likhterov B., Kopeika N.S, Differential Sensing of Vibration for High-Quality Restoration of Motion-Blurred Images. Optical Engineering, vol. 41, no. 11, 2970-2974, 2002.

Mammone R.J., Computation Methods of Signal Recovery and Recognition. John Wiley \& Sons, 1992.

Kuo, S.S., Mammone R.J., Image Restoration by Convex Projections Using Adaptive Constraints and the L1 Norm. IEEE Transactions on Signal Processing, vol. 40, 159-168, 1992.

Papa, J.P., Mascarenhas, N.D.A, Fonseca L.M.G., A Comparison between Projections Algorithms for CBERS-1 Satellite Image Restoration. XII Brazilian Simposium on Remote Sensing, 1045-1052, 2005.

Reichenbach, S.E. Koehler, D.E. and Strelow, D.W., Restoration and Recon- 
INPE ePrint: sid.inpe.br/ePrint@80/2006/10.24.19.25 v2 2007-06-05 REFERENCES

struction of AVHRR Images. IEEE Transactions on Geoscience and Remote Sensing, vol. 33, 997-1007, 1995.

Sezan M.I., Trussel, H.J., Prototype Image Constraints for Set-Theoretic Image Restoration. IEEE Transactions on Signal Processing, vol. 39, no. 10, 2275-2285, 1991.

Stark H., Yang, Y., Vector Space Projections. Wiley-Interscience 1998.

Wang Z., Bovik, A.C. A Universal Image Quality Index. IEEE Signal Processing Letters, vol. 9, no. 3, 81-8, 2002.

Wu H.-H.P., Schowengerdt, R.A., Improved Estimation of Fraction Images Using Partial Image Restoration. IEEE Transactions on Remote Sensing, vol. 31, no. 4, 771-778, 1993. 